\title{
ON RATIONAL NORMAL CURVES IN PROJECTIVE SPACE
}

\author{
E. CARLINI, M. V. CATALISANO
}

\begin{abstract}
In this paper we consider a generalization of a well known result by Veronese about rational normal curves. More precisely, given a collection of linear spaces in $\mathbb{P}^{n}$ we study the existence of rational normal curves intersecting each component of the configuration maximally. We introduce different methods to show existence and non-existence of such curves. We also show how to apply these techniques to the study of defectivity of Segre-Veronese varieties.
\end{abstract}

\section{Introduction}

The starting point of our study is the following well know result

Theorem 1.1 (Veronese). Given $n+3$ generic points in $\mathbb{P}^{n}$ there exists a unique rational normal curve passing through them.

Although often attributed to Castelnuovo, this theorem was proved by Veronese [Ver82]. Alternative proofs can be produced using a constructive approach as shown in [Har92, page 10] or via Cremona transformations as recalled in [CC07, Theorem 4.1]. In fact, a new proof can be given using the methods of this paper, see Remark 3.13 .

Veronese's result can be generalized in different ways. For example, one can consider more general varieties passing through points, e.g. $d$-uple embeddings of the projective plane. Kapranov, for example, studied a possible generalization of Theorem 1.1 to Veronese surfaces in [Kap93], see also [GR03] for another proof of Kapranov's result. A strictly related problem is treated in [EHP03] where the maximal number of intersection points of two Veronese surfaces is determined. In a different direction, one can still consider rational normal curves, but now satisfying more general conditions. In the early twentieth century, for example, some interest focused on rational normal curves passing through points and intersecting codimension two linear spaces of $\mathbb{P}^{n}$ in $n-1$ points, i.e. in the maximal number of points allowed, see [Roo38, page 221],[Wak22] and [Bab33]. In [CC07] we considered this precise situation and we were able to recover all the known results and to prove new ones. Our analysis yielded the following:

Theorem 1.2 (Theorem 4.7 in [CC07]). Let $n, p$ and $l$ be non-negative integers such that

$$
n \geq 3, \quad p \geq 1 \text { and } p+l=n+3 .
$$

Choose $p$ generic points in $\mathbb{P}^{n}$ and l generic codimension two linear spaces. Then, for the values

$$
(p, l)=(n+3,0),(n+2,1),(3, n),(2, n+1),(1, n+2)
$$

1991 Mathematics Subject Classification. 14H45; 14N20 . 
does there exist a unique rational normal curve passing through the points and $(n-1)$-secant to the linear spaces. In the other cases, that is for $p \geq 4$ and $l \geq 2$, no such curve exists.

As an application of this result, we studied higher secant deficiencies for some Segre-Veronese varieties, see [CC07]. For more on Segre-Veronese varieties see [CGG05, CGG07] and for more on defective varieties [Zak93, Ger96, Cil01]. In [CC07], we also noticed that rational normal curves satisfying more general condition could be useful, see Remark 3.12. More precisely, it is interesting and useful to consider the following question

given a finite collection of linear spaces in generic position in $\mathbb{P}^{n}$, is there a rational normal curve intersecting each $i$-dimensional component of the collection in $i+1$ points?

This paper is devoted to the study of this last question. Giving a complete answer appears to very ambitious and quite difficult and so our result are only partial. Nevertheless, they do give some understanding of the situation. For example, Corollary 4.2 shows that for any $n$ there are only finitely many cases to consider. While Theorem 4.8 and Proposition 4.4 produce an answer in some of the remaining cases. To understand the intrinsic difficulty of the question, we notice that even when all the spaces have dimension equal to one, there are open cases, see Subsection 5.1.

The relevant information to describe a generic collection of linear spaces $\Lambda=\cup \Lambda_{i}$ can be encoded in a vector of non-negative integers $L=\left(l_{0}, \ldots, l_{n-2}\right)$ where $l_{i}$ is the number of $i$-dimensional spaces in $\Lambda$. Now, given $L$, should we expect a positive or a negative answer to our question? We can develop some heuristic machinery to help our intuition. Using the matrix representation of a rational normal curve, one can easily see that the rational normal curves passing through a given point are a codimension $n-1$ family in their natural parameter space. The rational normal curves 2 -secant to a given line are a family of codimension $2(n-2)$. Similarly, the family of rational normal curves 3 -secant to a given plane has codimension $3(n-3)$. In conclusion, as the parameter space for rational normal curves in $\mathbb{P}^{n}$ has dimension $(n+3)(n-1)$, given $\Lambda$ with relevant vector $\left(l_{0}, \ldots, l_{n-2}\right)$, we expect there to be a family, of rational normal curves, which has dimension

$$
(n+3)(n-1)-\sum_{i=0}^{n-2} l_{i}(i+1)(n-1-i),
$$

to be $(i+1)$-secant to each $i$-dimensional component of $\Lambda$. This kind of argument will be made rigorous and used to derive results in Subsection 4.1. Since the question is not clearly linear in nature, the actual dimension of the family can very well be bigger or smaller than the expected one.

In Section 2, we introduce some notation for configurations of linear spaces and their relevant vectors.

In Section 3, we introduce methods to show existence of rational normal curves.

In Section 4, we describe methods to prove non-feasibility.

In Section 5, we apply the methods developed in the paper to study some special situations and to produce results about Segre-Veronese varieties and the behavior of their higher secant varieties. More precisely, we study homogeneous configurations, where all the components have the same dimension. In the simplified situation 
when all linear spaces have dimension equal to one, we have a complete answer except for five lines in $\mathbb{P}^{5}$ and six lines in $\mathbb{P}^{7}$, see Proposition 5.2. We also study the configurations consisting of exactly one space in each dimension, giving an answer for all cases, except for $\mathbb{P}^{6}$ and $\mathbb{P}^{7}$, see Proposition 5.3. We present a family of non-feasible configurations, not containing points, which stems from the analysis of the lines case. Finally, we show how to apply our results to find new defectivity results for the Segre-Veronese varieties $\mathbb{P}^{1} \times \mathbb{P}^{m} \times \mathbb{P}^{m}$ embedded with multi-degree $(2,1,1)$. For more on Segre-Veronese varieties see [CGG05, CGG07].

Remark 1.3. Our question seems to be suitable for an Intersection Theory approach and we posed the question to many experts in that field. However, as far as we know, there is no "ready-to-use-machinery" which can easily produce explicit results.

The authors wish to thank Gianfranco Casnati for stimulating further investigations on the map used in Subsection 3.2. We also want to acknowledge Tony Geramita's suggestion to look further into the lines case. His suggestion led to Subsection 5.1.

\section{Definition}

We work over the field of complex numbers $\mathbb{C}$. A rational normal curve in $\mathbb{P}^{n}$ will be denoted as an $r n c$ in $\mathbb{P}^{n}$.

A vector of non-negative integers $L=\left(l_{0}, \ldots, l_{n-2}\right)$ is called a weight vector in $\mathbb{P}^{n}$. A scheme $\Lambda \subset \mathbb{P}^{n}$ is said to be a configuration of linear spaces of weight $L$ if $\Lambda$ is the union of $l_{i}$ linear spaces of dimension $i$, for $i=0, \ldots, n-2$; for we will denote this configuration as $\Lambda_{L}$. We say that $\Lambda_{L}$ is generic if the linear components of $\Lambda$ are chosen in a suitable open non-empty subset of a product of Grassmannians.

Remark 2.1. There is a great deal of literature dealing with subspace arrangements and these are nothing more than the affine interpretation of what we call a configuration of linear spaces, e.g. see [Deb08],[BPS05] and [Sid07]. In particular, in [Der07] the Hilbert polynomial of a generic subspace arrangements is determined and this is a first step in the direction of the study of Hilbert function. This is relevant to us as the knowledge of the Hilbert function of a configuration of linear spaces is needed for the application of Proposition 4.4.

We say that a configuration of linear spaces $\Lambda=\Lambda_{1} \cup \cdots \cup \Lambda_{t} \subset \mathbb{P}^{n}$ is feasible if there exists a $\operatorname{rnc} \mathcal{C} \subset \mathbb{P}^{n}$ maximally intersecting it, i.e. if $\operatorname{deg}\left(\mathcal{C} \cap \Lambda_{i}\right)=\operatorname{dim} \Lambda_{i}+1$, for any component $\Lambda_{i}$ of $\Lambda$.

We say that the vector $L$ is feasible if and only if a generic configuration of linear spaces of weight $L$ is feasible.

Remark 2.2. With this notation we can rephrase Theorem 1.1as follows:

The weight vector $(p, 0, \ldots, 0)$ in $\mathbb{P}^{n}$ is feasible if and only if $p \leq$ $n+3$.

Similarly, Theorem 1.2 reads as follows:

The weight vector $(p, 0, \ldots, l), p \geq 1$, in $\mathbb{P}^{n}$ is feasible if and only if $p \leq 4$ or $l \leq 2$. 


\section{Methods FOR FEAsibiLity}

3.1. Veronese's Theorem. As a straightforward application of Theorem 1.1 we get a useful numerical condition for feasibility.

Proposition 3.1. Let $L=\left(l_{0}, \ldots, l_{n-2}\right)$ be a weight vector in $\mathbb{P}^{n}$. If

$$
\sum_{i=0}^{n-2}(i+1) l_{i} \leq n+3
$$

then $L$ is feasible.

Proof. Given a generic configuration of linear spaces $\Lambda_{L}$, choose $i+1$ generic points on each $i$-dimensional component of $\Lambda_{L}$ and apply Theorem 1.1.

Remark 3.2. Notice that the numerical condition of the previous Proposition is sufficient, but not necessary. Consider, for example, the weight vector $L=(5,1)$ in $\mathbb{P}^{3}$. By Theorem 1.2 we know that $L$ is feasible, see [CC07] for more details. However,

$$
\sum_{i=0}^{n-2}(i+1) l_{i}=7>n+3=6
$$

and feasibility can not be deduced by Proposition 3.1 .

3.2. Segre varieties. In this subsection, we use Segre varieties as a tool to verify feasibility. We begin with a study of Segre varieties and then we show how they are involved in the study of rncs. In particular, we start from the description of an explicit parametrization of these varieties which can be found in [CGG05, pg.84]. Then, we study the existence of rational curves with special properties on the Segre varieties. Coupling these results we can prove feasibility results.

We now fix notation for this section: for positive integers $n_{1} \leq n_{2} \ldots \leq n_{r}$, we let $n=n_{1}+n_{2} \ldots+n_{r}$. Consider the $n$ dimensional abstract Segre variety

$$
\mathbf{S}=\mathbb{P}^{n_{1}} \times \ldots \times \mathbb{P}^{n_{r}}
$$

and its Segre embedding

$$
\mathbf{S} \hookrightarrow \mathcal{S} \subset \mathbb{P}^{N}
$$

where $N=\left(n_{1}+1\right) \cdot \ldots \cdot\left(n_{r}+1\right)-1$.

Given hyperplanes

$$
H_{1} \subset \mathbb{P}^{n_{1}}, \ldots, H_{r} \subset \mathbb{P}^{n_{r}}
$$

we denote by $\mathbf{T}$ the Segre variety

$$
\mathbf{T}=H_{1} \times \ldots \times H_{r} \subset \mathbf{S}
$$

and by $\mathcal{I}_{\mathbf{T}}$ its ideal sheaf. Consider $\left(\mathcal{I}_{\mathbf{T}}\right)^{r-1}$ in multidegree $(1, \ldots, 1)$, let

$$
\mathcal{L}=H^{0}\left(\mathbf{S},\left(\mathcal{I}_{\mathbf{T}}\right)^{r-1}(1, \ldots, 1)\right)
$$

and let $\phi_{\mathbf{T}}$ be the standard map:

$$
\phi_{\mathbf{T}}: \mathbf{S} \rightarrow \mathbb{P}(\mathcal{L})^{*} .
$$

Recall that the Chow ring of the Segre variety is

$$
C H(\mathbf{S})=\frac{\mathbb{Z}\left[h_{1}, \ldots, h_{r}\right]}{\left(h_{1}^{n_{1}+1}, \ldots, h_{r}^{n_{r}+1}\right)},
$$


where the classes $h_{i}$ are the pull backs of the hyperplane classes via the standard projection maps

$$
\pi_{i}: \mathbf{S} \longrightarrow \mathbb{P}^{n_{i}} .
$$

Let

$$
\Lambda_{i}=\phi_{\mathbf{T}}\left(\pi_{i}^{-1}\left(H_{i}\right)\right) \subset \mathbb{P}^{n}, \quad(i=1, \ldots, r) .
$$

Lemma 3.3. The map $\phi_{\mathbf{T}}$ is a birational map,

$$
\operatorname{dim}_{\mathbb{C}} \mathcal{L}=n+1
$$

where $n=\sum n_{i}$, and the indeterminacy locus is

$$
\mathbf{B}=\bigcup_{1 \leq i<j \leq r} \pi_{i}^{-1}\left(H_{i}\right) \cap \pi_{j}^{-1}\left(H_{j}\right) .
$$

Proof. Consider the coordinate ring of the Segre variety $\mathbf{S}$

$$
R=\mathbb{C}\left[x_{0}^{(1)}, \ldots, x_{n_{1}}^{(1)}, x_{0}^{(2)}, \ldots, x_{n_{2}}^{(2)}, \ldots, x_{0}^{(r)}, \ldots, x_{n_{r}}^{(r)}\right]
$$

and assume that $H_{i}=\left\{x_{0}^{(i)}=0\right\}$, for $1 \leq i \leq r$. Then we have that $\mathcal{L}$ is the multidegree $(1, \ldots, 1)$ part of the ideal

$$
\left(x_{0}^{(1)}, \ldots, x_{0}^{(r)}\right)^{r-1} \subset R,
$$

which is generated by $n+1$ linear independent forms

$$
\begin{gathered}
x_{0}^{(1)} \cdot \ldots \cdot x_{0}^{(r)}, \\
x_{1}^{(1)} x_{0}^{(2)} \cdot \ldots \cdot x_{0}^{(r)}, \ldots, x_{n_{1}}^{(1)} x_{0}^{(2)} \cdot \ldots \cdot x_{0}^{(r)}, \\
\vdots \\
x_{0}^{(1)} \cdot \ldots \cdot x_{0}^{(r-1)} x_{1}^{(r)}, \ldots, x_{0}^{(1)} \cdot \ldots \cdot x_{0}^{(r-1)} x_{n_{r}}^{(r)} .
\end{gathered}
$$

Hence the base locus of $\mathcal{L}$ is the collection of points $Q_{1} \times \ldots \times Q_{r}$ such that at least two of them, say $Q_{i} \in \mathbb{P}^{n_{i}}$ and $Q_{j} \in \mathbb{P}^{n_{j}}$, lie on the hyperplanes $H_{i}$ and $H_{j}$, respectively. Thus the base locus is

$$
\mathbf{B}=\bigcup_{1 \leq i<j \leq r} \pi_{i}^{-1}\left(H_{i}\right) \cap \pi_{j}^{-1}\left(H_{j}\right)
$$

Following [CGG05], for $Q \in \mathbf{S} \backslash \mathbf{B}$, we may write the map $\phi_{\mathbf{T}}$ as

$$
\begin{aligned}
\phi_{\mathbf{T}}(Q)= & {\left[x_{0}^{(1)} \cdot \ldots \cdot x_{0}^{(r)}, x_{1}^{(1)} x_{0}^{(2)} \cdot \ldots \cdot x_{0}^{(r)}, \ldots, x_{n_{1}}^{(1)} x_{0}^{(2)} \cdot \ldots \cdot x_{0}^{(r)}, \ldots,\right.} \\
& \left.x_{0}^{(1)} \cdot \ldots \cdot x_{0}^{(r-1)} x_{1}^{(r)}, \ldots, x_{0}^{(1)} \cdot \ldots \cdot x_{0}^{(r-1)} x_{n_{r}}^{(r)}\right] .
\end{aligned}
$$

Hence for points

$$
Q=Q_{1} \times \ldots \times Q_{r} \in \mathbf{S} \backslash \bigcup_{i=1}^{r} \pi_{i}^{-1}\left(H_{i}\right),
$$

if we let $Q_{i}=\left[1, q_{1}^{(i)}, \ldots, q_{n_{i}}^{(i)}\right]$, the map $\phi_{\mathbf{T}}$ can be nicely written as

$$
\phi_{\mathbf{T}}(Q)=\left[1, q_{1}^{(1)}, \ldots, q_{n_{i}}^{(1)}, q_{1}^{(2)}, \ldots, q_{n_{r-1}}^{(r-1)}, q_{1}^{(r)}, \ldots, q_{n_{r}}^{(r)}\right],
$$

and this is enough to show that the map is birational (see [CGG05] for more details). 
Lemma 3.4. There exists a linear space $\Sigma \subset \mathbb{P}^{N}$ such that, for a suitable choice of the basis of $\mathcal{L}, \phi_{\mathbf{T}}$ is induced by the projection from $\Sigma$, that is we have a commutative diagram

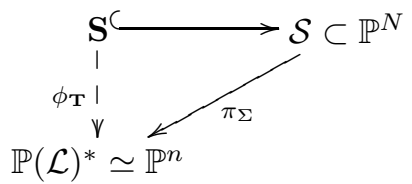

where $\pi_{\Sigma}$ denotes the projection map from $\Sigma$. Moreover, $\Sigma \cap \mathcal{S}$ is the Segre embedding of $\mathbf{B}$.

Proof. By rearranging the forms of (1) in lexicographic order, we write the map $\phi_{\mathbf{T}}$ as

$$
\begin{aligned}
\phi_{\mathbf{T}}(Q)= & {\left[x_{0}^{(1)} \cdot \ldots \cdot x_{0}^{(r)}, x_{0}^{(1)} \cdot \ldots \cdot x_{0}^{(r-1)} x_{1}^{(r)}, \ldots, x_{0}^{(1)} \cdot \ldots \cdot x_{0}^{(r-1)} x_{n_{r}}^{(r)}, \ldots,\right.} \\
& \left.x_{1}^{(1)} x_{0}^{(2)} \cdot \ldots \cdot x_{0}^{(r)}, \ldots, x_{n_{1}}^{(1)} x_{0}^{(2)} \cdot \ldots \cdot x_{0}^{(r)}\right]
\end{aligned}
$$

where $Q \in \mathbf{S} \backslash \mathbf{B}$. As the Segre embedding $\mathbf{S} \hookrightarrow \mathcal{S} \subset \mathbb{P}^{N}$ is defined by all the multidegree $(1, \ldots, 1)$ forms in the coordinate ring $R$, it is enough to consider

$$
\Sigma=\left\{x_{0}^{(1)} \cdot \ldots \cdot x_{0}^{(r)}=x_{1}^{(1)} x_{0}^{(2)} \cdot \ldots \cdot x_{0}^{(r)}=\ldots=x_{0}^{(1)} \cdot \ldots \cdot x_{0}^{(r-1)} x_{n_{r}}^{(r)}=0\right\} \subset \mathbb{P}^{N} .
$$

The computation of $\Sigma \cap \mathcal{S}$ is straightforward.

As we know that $\phi_{\mathbf{T}}$ is a birational map it is interesting to investigate subvarieties which are contracted by this map.

Lemma 3.5. The space

$$
\Lambda_{i}=\phi_{\mathbf{T}}\left(\pi_{i}^{-1}\left(H_{i}\right)\right) \subset \mathbb{P}^{n},(i=1, \ldots, r)
$$

is a $n_{i}-1$ dimensional linear space. Moreover, $\Lambda_{1}, \ldots, \Lambda_{r}$ are generic linear spaces in $\mathbb{P}^{n}$.

Proof. As in the proof of Lemma 3.3, we assume that $\pi_{i}^{-1}\left(H_{i}\right)$ is defined by $\left\{x_{0}^{(i)}=\right.$ $0\}$ on the Segre variety, and that $\phi_{\mathbf{T}}$ is as in (1).

Consider the coordinate ring of $\mathbb{P}^{n}$

$$
\mathbb{C}\left[y_{0}, y_{1}^{(1)}, \ldots, y_{n_{1}}^{(1)}, \ldots, y_{1}^{(r)}, \ldots, y_{n_{r}}^{(r)}\right] .
$$

We have that

$\Lambda_{1}$ is defined by $\left\{y_{0}=y_{1}^{(2)}=\ldots=y_{n_{2}}^{(2)}=\ldots=y_{1}^{(r)}=\ldots=y_{n_{r}}^{(r)}=0\right\}$,

$\Lambda_{r}$ is defined by $\left\{y_{0}=y_{1}^{(1)}=\ldots=y_{n_{1}}^{(1)}=\ldots=y_{1}^{(r-1)}=\ldots=y_{n_{r-1}}^{(r-1)}=0\right\}$.

Thus, the spaces $\Lambda_{i}$ are linear spaces of dimension $n_{i}-1$. Notice that each $\Lambda_{i}$ is the span of a set of coordinate points and that each of these coordinate points only belongs to one $\Lambda_{i}$. Hence the spaces $\Lambda_{i}$ are generic linear spaces in $\mathbb{P}^{n}$.

To bring rational curves into the picture, we recall a rather elementary technical fact (see [Har92, page 10]).

Lemma 3.6. Let $A_{1}, \ldots, A_{m} \in \mathbb{P}^{1}$ and $Q_{1}, \ldots, Q_{m} \in \mathbb{P}^{t}$ be generic points. If $m \leq t+2$, then there exists a morphism $\psi: \mathbb{P}^{1} \longrightarrow \mathbb{P}^{t}$ whose image is a rational normal curve of degree $t$ and such that $\psi\left(A_{i}\right)=Q_{i}, i=1, \ldots, m$. 
Proof. It is enough to give the proof for $m=t+2$. For smaller $m$ we can just add some extra points and repeat the same argument. After a projectivity, we may assume that $Q_{1}=[1,0, \ldots, 0], Q_{2}=[0,1,0 \ldots, 0], \ldots, Q_{t+1}=[0, \ldots, 0,1]$ and $Q_{t+2}=[1, \ldots, 1]$. Then, we choose $t+1$ linear forms $L_{1}, \ldots, L_{t+1}$ on $\mathbb{P}^{1}$ such that $L_{i}\left(A_{i}\right)=0, \quad(1 \leq i \leq t+1)$, and we consider the map

$$
\psi: \mathbb{P}^{1} \longrightarrow \mathbb{P}^{t}
$$

defined for $P \notin\left\{A_{1}, \ldots, A_{t+1}\right\}$ by

$$
P \mapsto\left[\frac{a_{1}}{L_{1}(P)}, \ldots, \frac{a_{i}}{L_{i}(P)}, \ldots, \frac{a_{t+1}}{L_{t+1}(P)}\right],
$$

where $a_{i}=L_{i}\left(A_{t+2}\right),(1 \leq i \leq t+1)$, and naturally extended. It is easy to check that $\psi$ is the required map.

The previous lemma allows us to construct rational curves with some special properties on Segre varieties.

Proposition 3.7. Notation as above, let $n_{1} \leq n_{2} \ldots \leq n_{r}$ be positive integers, and let $\mathbb{X}$ be a set of $s$ generic points on the Segre variety

$$
\mathbf{S}=\mathbb{P}^{n_{1}} \times \ldots \times \mathbb{P}^{n_{r}} .
$$

i) If $s \leq n_{1}+3$ and $1<n_{1}<n_{2}$, or $s \leq n_{2}+2$ and $n_{1}=1$ or $n_{1}=n_{2}$, then there exists a rational curve $\mathbf{C}$ such that

$$
\mathbb{X} \subset \mathbf{C} \subset \mathbf{S}
$$

and $h_{i}[\mathbf{C}]=n_{i}$ in the Chow ring $C H(\mathbf{S})$.

ii) Let $\mathcal{C}$ be the Segre embedding of $\mathbf{C}$, then $\operatorname{deg}(\mathcal{C})=n=\sum n_{i}$ and the linear span of the curve is a $\mathbb{P}^{n}$, i.e. $\mathcal{C}$ is a rational normal curve in its linear span.

iii)

$$
\operatorname{deg}\left(\Lambda_{i} \cap \phi_{\mathbf{T}}(\mathbf{C})\right)=\operatorname{dim} \Lambda_{i}+1 .
$$

iv) $\phi_{\mathbf{T}}(\mathbf{C})$ is a rational normal curve of $\mathbb{P}^{n}$.

Proof. i) Let $t$ denote the maximum number of points such that there exists an rnc in $\mathbb{P}^{n_{1}}$ through $t$ generic points, that is $t=n_{1}+3$ if $n_{1}>1$ (see Theorem 1.1), and $t=\infty$ if $n_{1}=1$. Let $\bar{s}=\min \left\{t, n_{2}+2\right\}$. We have

$$
\bar{s}=\min \left\{t, n_{2}+2\right\}=\left\{\begin{array}{ll}
n_{1}+3 & \text { for } 1<n_{1}<n_{2} \\
n_{2}+2 & \text { for } n_{1}=1 \text { or } n_{1}=n_{2}
\end{array},\right.
$$

and it suffices to prove the proposition for $s=\bar{s}$.

Let $\mathbb{X}=\left\{P_{1}, \ldots, P_{\bar{s}}\right\}$ where

$$
P_{i}=Q_{i}^{(1)} \times \ldots \times Q_{i}^{(r)}, \quad\left(Q_{i}^{(j)} \in \mathbb{P}^{n_{j}}\right)
$$

and the points $Q_{1}^{(j)}, \ldots, Q_{\bar{s}}^{(j)}$ are generic in $\mathbb{P}^{n_{j}}$.

Since $\bar{s} \leq t$, there exists an embedding

$$
\psi_{1}: \mathbb{P}^{1} \hookrightarrow \mathbb{P}^{n_{1}}
$$

whose image is a rational normal curve passing through the points $Q_{1}^{(1)}, \ldots, Q_{\bar{s}}^{(1)}$. Observe that if $n_{1}=1$, then we can choose the identity map as $\psi_{1}$.

Let $A_{i}=\psi_{1}^{-1}\left(Q_{i}^{(1)}\right),(i=1, \ldots, \bar{s})$. Since $\bar{s} \leq n_{j}+2$ for any $j \geq 2$, by Lemma 3.6 we construct embeddings

$$
\psi_{j}: \mathbb{P}^{1} \hookrightarrow \mathbb{P}^{n_{j}}, j=2, \ldots, r
$$


such that $\psi_{j}\left(A_{i}\right)=Q_{i}^{(j)}$. Now we consider the curve $\mathbf{C}$ given as the image of the product map

$$
\psi_{1} \times \ldots \times \psi_{r}: \mathbb{P}^{1} \longrightarrow \mathbf{S}
$$

To compute $h_{i}[\mathbf{C}]$ in the Chow ring of $\mathbf{S}$, we recall that $h_{i}=\left[\pi_{i}^{-1}\left(H_{i}\right)\right]$ where $H_{i}$ is a hyperplane in $\mathbb{P}^{n_{i}}$. Then $h_{i}[\mathbf{C}]$ is the degree of $\pi_{i}(\mathbf{C})=\operatorname{Im}\left(\psi_{i}\right) \subset \mathbb{P}^{n_{i}}$ and this is $n_{i}$.

If we let $\mathcal{C} \subset \mathbb{P}^{N}$ be the Segre embedding of $\mathbf{C}$, then $\operatorname{deg}(\mathcal{C})$ can be determined by computing in $C H(\mathbf{S})$, i.e. we have to evaluate

$$
\left(h_{1}+\ldots+h_{r}\right)[\mathbf{C}]=\sum h_{i}[\mathbf{C}]=\sum n_{i}=n .
$$

ii) To show that the linear span $\langle\mathcal{C}\rangle$ of $\mathcal{C}$ has dimension $n$, recall that the curve $\mathcal{C}$ is the Segre embedding of $\mathbf{C}$, and that, for each $i, \psi_{i}\left(\mathbb{P}^{1}\right)$ is a rational normal curve. Now let $\mathrm{R}=\mathbb{C}[x, y]$ be the coordinate ring of $\mathbb{P}^{1}$, then $\psi_{i}\left(\mathbb{P}^{1}\right)$ is the image of the linear system $\mathrm{R}_{n_{i}}$ of the forms of degree $n_{i}$, and the multilinear multiplication map

is obviously surjective.

$$
\mathrm{R}_{n_{1}} \times \ldots \times \mathrm{R}_{n_{r}} \longrightarrow \mathrm{R}_{n_{1}+\ldots+n_{r}}=\mathrm{R}_{n}
$$

iii) As $\Lambda_{i}=\phi_{\mathbf{T}}\left(\pi_{i}^{-1}\left(H_{i}\right)\right)$ we can work in the Chow ring of $\mathbf{S}$ where

$$
\left[\Lambda_{i} \cap \phi_{\mathbf{T}}(\mathbf{C})\right]=h_{i}[\mathbf{C}]=n_{i}=\operatorname{dim} \Lambda_{i}+1 .
$$

iv) To show that $\phi_{\mathbf{T}}(\mathbf{C})$ is an rnc it is enough to prove that

$$
\langle\mathcal{C}\rangle \cap \Sigma=\emptyset,
$$

or, equivalently, to prove that $\phi_{\mathbf{T}}(\mathbf{C})$ does not lie on a hyperplane, i.e. it is not degenerate.

Assume that $\phi_{\mathbf{T}}(\mathbf{C})$ lies on a hyperplane $H$. Since $\mathbb{X}$ is a set of generic points, then $H \neq\left\langle\Lambda_{1}, \ldots, \Lambda_{r}\right\rangle$, hence there exists at least one $\Lambda_{i}$ such that the points of

$$
\Lambda_{i} \cap \phi_{\mathbf{T}}(\mathbf{C})
$$

are degenerate in $\Lambda_{i}$, and this is the case if and only if the points of

$$
H_{i} \cap \operatorname{Im}\left(\psi_{i}\right)
$$

are degenerate in $H_{i}$, in fact, by (2), the spaces $\Lambda_{i}$ are naturally identified with the hyperplanes $H_{i}$. Since $\operatorname{Im}\left(\psi_{i}\right)$ is an rnc, we get a contradiction.

Remark 3.8. Note that for $n_{1}=1$ the proof of the previous proposition does not require Veronese's Theorem.

Remark 3.9. We give a sketch of an alternative proof of Proposition 3.7 iv).

With the notation as above, let $Q$ be a point in $\mathbb{P}^{n_{j}}$ and set

$$
\psi_{j}(Q)=\left(\left.f_{0}^{(j)}\right|_{Q}, \ldots,\left.f_{n_{j}}^{(j)}\right|_{Q}\right) \in \mathbb{P}^{n_{j}},
$$

where $f_{0}^{(j)}, \ldots, f_{n_{j}}^{(j)}$ is a base of $R_{n_{j}}$. Then by (1) we get that the curve $\phi_{\mathbf{T}}(\mathbf{C})$ is the image of the linear system:

$$
\begin{gathered}
\left(f_{0}^{(1)} \cdot \ldots \cdot f_{0}^{(r)}, f_{1}^{(1)} f_{0}^{(2)} \cdot \ldots \cdot f_{0}^{(r)}, \ldots, f_{n_{1}}^{(1)} f_{0}^{(2)} \cdot \ldots \cdot f_{0}^{(r)}, \ldots,\right. \\
\left.f_{0}^{(1)} \cdot \ldots \cdot f_{0}^{(r-1)} f_{1}^{(r)}, \ldots, f_{0}^{(1)} \cdot \ldots \cdot f_{0}^{(r-1)} f_{n_{r}}^{(r)}\right) \subset \mathrm{R}_{n} .
\end{gathered}
$$

If we prove that these $n+1$ forms are linearly independent, then we are done. 
The key point to do this is the following: there does not exist a point $A \in \mathbb{P}^{1}$ such that $\left.f_{0}^{(i)}\right|_{A}=\left.f_{0}^{(j)}\right|_{A}=0$, for $i \neq j$, and this is a (non-obvious) consequence of the genericity of $\mathbb{X}$.

Now our goal is to use the curves of the proposition above to address the problem of feasibility.

Theorem 3.10. Let $n_{1} \leq \ldots \leq n_{r}$ be positive integers, and set $n=n_{1}+\ldots+n_{r}$. Consider in $\mathbb{P}^{n}$ a set of $s$ generic points $P_{1}, \ldots, P_{s}$ and $r$ generic linear spaces $\Lambda_{1}, \ldots, \Lambda_{r}$ of dimension $n_{1}-1, \ldots, n_{r}-1$, respectively.

If $s \leq n_{1}+3$ and $1<n_{1}<n_{2}$, or $s \leq n_{2}+2$ and $n_{1}=1$ or $n_{1}=n_{2}$, then the configuration of linear spaces

$$
\Lambda_{1} \cup \ldots \cup \Lambda_{r} \cup\left\{P_{1}, \ldots, P_{s}\right\} \subset \mathbb{P}^{n}
$$

is feasible.

Proof. Consider the Segre variety $\mathbb{P}^{n_{1}} \times \cdots \times \mathbb{P}^{n_{r}}$ and choose hyperplanes $H_{i} \subset \mathbb{P}^{n_{i}}$ such that the birational map

$$
\phi_{\mathbf{T}}: \mathbb{P}^{n_{1}} \times \cdots \times \mathbb{P}^{n_{r}} \rightarrow \mathbb{P}^{n},
$$

where $\mathbf{T}=H_{1} \times \ldots \times H_{r}$, contracts

$$
\begin{gathered}
H_{1} \times \mathbb{P}^{n_{2}} \times \cdots \times \mathbb{P}^{n_{r}}, \\
\mathbb{P}^{n_{1}} \times H_{2} \times \mathbb{P}^{n_{3}} \times \cdots \times \mathbb{P}^{n_{r}}, \\
\vdots \\
\mathbb{P}^{n_{1}} \times \cdots \times \mathbb{P}^{n_{r-1}} \times H_{r}
\end{gathered}
$$

onto the linear spaces $\Lambda_{1}, \ldots, \Lambda_{r}$, respectively.

Chosen generic points $P_{1}, \ldots, P_{s} \in \mathbb{P}^{n}$, via the map $\phi_{\mathbf{T}}$ these points correspond to $s$ generic points on the Segre variety. Then, the image of the curve of Proposition 3.7 is an rnc in $\mathbb{P}^{n}$ passing through the points $P_{i}$, and $n_{i}-1$-secant to $\Lambda_{i}$. Hence, the generic configuration of linear spaces

$$
\Lambda_{1} \cup \ldots \cup \Lambda_{r} \cup\left\{P_{1}, \ldots, P_{s}\right\} \subset \mathbb{P}^{n}
$$

is feasible.

Example 3.11. We illustrate our strategy explicitly by studying the weight vector $L=(5,1,1,0)$ in $\mathbb{P}^{5}$. Let $P_{1}, \ldots, P_{5} \in \mathbb{P}^{5}$ be generic points, and $\Lambda_{1}, \Lambda_{2} \subset \mathbb{P}^{5}$ be generic linear spaces, of dimension 1 and 2 , respectively. Consider the Segre variety $\mathbb{P}^{2} \times \mathbb{P}^{3}$ and let $\mathbf{T} \subset \mathbb{P}^{2} \times \mathbb{P}^{3}, \mathbf{T}=H_{1} \times H_{2} \simeq \mathbb{P}^{1} \times \mathbb{P}^{2}$ be such that the birational map

$$
\phi_{\mathbf{T}}: \mathbb{P}^{2} \times \mathbb{P}^{3} \rightarrow \mathbb{P}^{5}
$$

contracts $H_{1} \times \mathbb{P}^{3}$, and $\mathbb{P}^{2} \times H_{2}$, onto the line $\Lambda_{1}$, and the plane $\Lambda_{2}$, respectively. Via the map $\phi_{\mathbf{T}}$, the points $P_{i}$ corresponds to 5 generic points in $\mathbb{P}^{2} \times \mathbb{P}^{3}$. So the image, via the map $\phi_{\mathbf{T}}$, of the curve in Proposition 3.7 is an rnc in $\mathbb{P}^{5}$ passing through the points $P_{i}, 2$-secant to $\Lambda_{1}$ and 3 -secant to $\Lambda_{2}$. Hence $L$ is feasible.

Remark 3.12. The previous example is related to the study of the variety of 5secant $\mathbb{P}^{4}$ 's to the Segre-Veronese variety $\mathbb{P}^{2} \times \mathbb{P}^{3}$ embedded using forms of bidegree $(1,2)$. As noticed in [CC07, Subsection 6.2], the existence of the rnc yields that this Segre-Veronese variety is 5 -defective. 
Remark 3.13. We notice that Theorem 3.10 and Remark 3.8 allow us to recover Veronese's Theorem in $\mathbb{P}^{n}$. It is enough to consider $r=n, n_{1}=\ldots=n_{r}=1$, and $s=3$ to show that the weight vector $(n+3,0, \ldots, 0)$ in $\mathbb{P}^{n}$ is feasible.

Remark 3.14. Another proof of the feasibility of $(n+2,0, \ldots, 0,1)$ in $\mathbb{P}^{n}$ can be deduced by Theorem 3.10 with $r=2, n_{1}=1, n_{2}=n-1$, and $s=n_{2}+2=n+1$ generic points (see also Theorem 1.2).

\section{Methods For NON-FEASIBILITy}

4.1. Parameter space. We can use a natural parameter space for rncs to get a powerful non-feasibility condition. Given a weight vector $L$ in $\mathbb{P}^{n}$ we consider a parameter space for the configurations of linear spaces $\Lambda_{L}$, namely

$$
\mathcal{D}=\left(G\left(\mathbb{P}^{0}, \mathbb{P}^{n}\right)\right)^{l_{0}} \times \ldots \times\left(G\left(\mathbb{P}^{i}, \mathbb{P}^{n}\right)\right)^{l_{i}} \times \ldots \times\left(G\left(\mathbb{P}^{n-2}, \mathbb{P}^{n}\right)\right)^{l_{n-2}},
$$

where $G\left(\mathbb{P}^{i}, \mathbb{P}^{n}\right)$ is the Grassmannian of the $i$-dimensional linear subspaces of $\mathbb{P}^{n}$. We also recall that there exists an irreducible component of the Hilbert scheme, namely

$$
\mathcal{H} \subset \operatorname{Hilb}_{n t+1}\left(\mathbb{P}^{n}\right)
$$

which is a parameter spaces for rncs in $\mathbb{P}^{n}$. Finally, we consider the incidence correspondence

$$
\mathcal{H} \times \mathcal{D} \supset \Sigma=\left\{\left(\mathcal{C}, \Lambda_{L}\right): \mathcal{C} \text { maximally intersects } \Lambda_{L}\right\}
$$

and the natural projection map $\psi: \Sigma \longrightarrow \mathcal{D}$. Clearly we have that $L$ is feasible if and only if $\psi$ is a dominant map.

Proposition 4.1. Let $L=\left(l_{0}, \ldots, l_{n-2}\right)$ be a weight vector in $\mathbb{P}^{n}$. If

$$
\sum_{i=0}^{n-2}(i+1)(n-1-i) l_{i}>(n+3)(n-1),
$$

then $L$ is non-feasible.

Proof. With the notation as above, we compute $\operatorname{dim} \Sigma$. In order to do this, we use the projection $\Sigma \longrightarrow \mathcal{H}$ whose fibers have dimension

$$
l_{0}+2 l_{1}+\ldots+(i+1) l_{i}+\ldots+(n-1) l_{n-2}=\sum_{i=0}^{n-2}(i+1) l_{i} .
$$

As $\operatorname{dim} \mathcal{H}=(n+3)(n-1)$, we get

$$
\operatorname{dim} \Sigma=(n+3)(n-1)+\sum_{i=0}^{n-2}(i+1) l_{i} .
$$

Now we compute $\operatorname{dim} \Sigma-\operatorname{dim} \mathcal{D}$. We have:

$$
\begin{gathered}
\operatorname{dim} \Sigma-\operatorname{dim} \mathcal{D}=(n+3)(n-1)+\sum_{i=0}^{n-2}(i+1) l_{i}-\sum_{i=0}^{n-2}(i+1)(n-i) l_{i} \\
=(n+3)(n-1)-\sum_{i=0}^{n-2}(i+1)(n-1-i) l_{i}<0
\end{gathered}
$$

Thus $\psi$ is not a dominant map, and $L$ is non-feasible. 
A priori, there are infinitely many weight vectors that one should study for feasibility in each $\mathbb{P}^{n}$, but from the previous proposition we immediately get the following

Corollary 4.2. There are only finitely many feasible weight vectors in $\mathbb{P}^{n}$.

Remark 4.3. The numerical conditions of the Proposition above is sufficient, but it is not necessary for feasibility. For instance consider the weight vector $L=(4,2)$ in $\mathbb{P}^{3}$. By Theorem 1.2 we know that $L$ is non-feasible, see also [CC07]. However, one has

$$
\sum_{i=0}^{n-2}(i+1)(n-1-i) l_{i}=12=(n+3)(n-1)
$$

and non-feasibility can not be obtained by Proposition 4.1.

4.2. Bezout argument. A simple elaboration of a Bezout type argument provides a useful tool to prove non-feasibility in many interesting situations, for example when Proposition 4.1 cannot be applied.

The starting point is a consequence of the Riemann-Roch Theorem for rational curves. Namely, given points $P_{i}$ with multiplicity $m_{i}$, and a degree $n$ very ample divisor $H$ on $\mathbb{P}^{1}$ one has

$$
h^{0}\left(\mathcal{O}_{\mathbb{P}^{1}}\left(d H-\sum m_{i} P_{i}\right)\right)=n d+1-\sum m_{i} .
$$

Hence, a degree $d$ hypersurface meeting a degree $n$ rnc in more than $n d$ points, counted with multiplicity, must contain the curve.

Proposition 4.4. Let $\Lambda^{\prime} \subset \mathbb{P}^{n}$ be a generic configuration of linear spaces, and let $\Gamma \subset \mathbb{P}^{n}$ be a generic $k$-dimensional linear space. Construct the configuration $\Lambda=\Lambda^{\prime} \cup \Gamma$ and let $L=\left(l_{0}, \ldots, l_{n-2}\right)$ be its weight vector. If, for an integer $d$, the following Hilbert function equality holds

$$
H(\Lambda, d)=H\left(\Lambda^{\prime}, d\right)+\left(\begin{array}{c}
d+k \\
k
\end{array}\right)
$$

and moreover

$$
\sum_{i=0}^{n-2} l_{i}(i+1)-1>d n
$$

then $L$ is non-feasible.

Proof. Assume that $\Lambda$ is feasible and let $\mathcal{C}$ be a required rnc. Consider the degree $k+1$ scheme $\mathcal{C} \cap \Gamma$ which, by the genericity of $\Lambda$, is reduced and can be written as

$$
\mathcal{C} \cap \Gamma=\left\{P_{1}, \ldots, P_{k+1}\right\} .
$$

Since the $k+1$ points $P_{i}$ are in linear general position, if we choose $t=\left(\begin{array}{c}d+k \\ k\end{array}\right)-(k+1)$ generic points $Q_{i} \in \Gamma$, then any hypersurface of degree $d$ passing through the $\left(\begin{array}{c}d+k \\ k\end{array}\right)$ points $P_{1}, \ldots, P_{k+1}, Q_{1}, \ldots, Q_{t}$ has $\Gamma$ as fixed component. Hence

$$
\operatorname{dim}\left(I_{\Lambda^{\prime} \cup P_{1} \cup \ldots \cup P_{k} \cup Q_{1} \cup \ldots \cup Q_{t}}\right)_{d}=\operatorname{dim}\left(I_{\Lambda^{\prime} \cup \Gamma}\right)_{d}=\operatorname{dim}\left(I_{\Lambda}\right)_{d}=\operatorname{dim}\left(I_{\Lambda^{\prime}}\right)_{d}-\left(\begin{array}{c}
d+k \\
k
\end{array}\right) .
$$


It follows that the $k+1$ points $P_{i}$ impose independent conditions to the forms of $\left(I_{\Lambda^{\prime}}\right)_{d}$. Now choose $k$ of them, say $P_{1}, \ldots, P_{k}$, let $F \in\left(I_{\Lambda^{\prime} \cup P_{1} \cup \ldots \cup P_{k}}\right)_{d}$ and notice that

$$
\operatorname{deg}(\{F=0\} \cap \mathcal{C})=\sum_{i=0}^{n-2} l_{i}(i+1)-(k+1)+k>d n,
$$

hence $\{F=0\} \supset \mathcal{C}$. Since $P_{k+1} \in \mathcal{C}$, we get

$$
\left(I_{\Lambda^{\prime} \cup P_{1} \cup \ldots \cup P_{k}}\right)_{d}=\left(I_{\Lambda^{\prime} \cup P_{1} \cup \ldots \cup P_{k} \cup P_{k+1}}\right)_{d}
$$

and this is a contradiction.

Example 4.5. Proposition 4.4 provides the non-feasibility in $\mathbb{P}^{n}$ of the weight vector $L=(4,0, \ldots, 0,2)$ using $k=0$, that is $\Gamma$ is a point, and $d=2$ (see also Theorem 1.2).

4.3. Projection argument. We present a simple argument which allows us to somehow improve Theorem 3.10 into a necessary and sufficient statement.

Lemma 4.6. Let $\Lambda \subset \mathbb{P}^{n}$ be a configuration of linear spaces. Let $\Lambda_{i}$ be an $i$ dimensional component of $\Lambda$, consider the projection $\pi$ from $\Lambda_{i}$

$$
\pi: \mathbb{P}^{n} \longrightarrow \mathbb{P}^{n-i-1}
$$

and let $\Lambda^{\prime}=\pi(\Lambda)$ be the projected configuration. If $\Lambda^{\prime}$ is non-feasible, then $\Lambda$ is non-feasible also.

Proof. Assume $\Lambda$ is feasible and let $\mathcal{C}$ be an rnc with the required properties. Then, $\pi(\mathcal{C})$ is a rational normal curve of $\mathbb{P}^{n-i}$ maximally intersecting $\Lambda^{\prime}$, a contradiction.

Remark 4.7. If the notation is as in the lemma above, notice that if $\Lambda$ is generic then $\Lambda^{\prime}$ is also. In particular, the conclusion can be read in term of weight vectors.

Theorem 4.8. Let $n_{1} \leq \ldots \leq n_{r}$ be positive integers, and set $n=n_{1}+\ldots+n_{r}$. Consider in $\mathbb{P}^{n}$ a set of $s$ generic points $P_{1}, \ldots, P_{s}$ and $r$ generic linear spaces $\Lambda_{1}, \ldots, \Lambda_{r}$ of dimension $n_{1}-1, \ldots, n_{r}-1$, respectively.

The configuration of linear spaces

$$
\Lambda=\Lambda_{1} \cup \ldots \cup \Lambda_{r} \cup\left\{P_{1}, \ldots, P_{s}\right\}
$$

is feasible if and only if $s \leq n_{1}+3$ and $1<n_{1}<n_{2}$, or $s \leq n_{2}+2$ and $n_{1}=1$ or $n_{1}=n_{2}$.

Proof. By Theorem 3.10, we only have to show that the numerical conditions on $s$ are necessary, more precisely, we have to prove that if $s \geq n_{1}+4$, or $s \geq n_{2}+3$ and $n_{1}=1$ or $n_{1}=n_{2}$, then $\Lambda$ is non-feasible.

Case: $s \geq n_{1}+4$. Consider the projection from the linear span

$$
\left\langle\Lambda_{2} \cup \ldots \cup \Lambda_{r}\right\rangle
$$

mapping $\mathbb{P}^{n}$ onto $\Pi_{1} \simeq \mathbb{P}^{n_{1}}$ and the $s$ points onto $s$ generic points in $\Pi_{1}$. Since in $\mathbb{P}^{n_{1}}$ there is not an rnc through $s \geq n_{1}+4$ generic points, we conclude by Lemma 4.6.

Case: $s \geq n_{2}+3$ and $n_{1}=1$. In this case, $\Lambda_{1}$ is a point, say $P$. Consider the projection from the linear space

$$
\left\langle\Lambda_{3} \cup \ldots \cup \Lambda_{r}\right\rangle,
$$


whose dimension is $\left(n_{3}+\ldots+n_{r}\right)=n-n_{2}-1$, mapping $\mathbb{P}^{n}$ onto $\Pi_{2} \simeq \mathbb{P}^{n_{2}+1}$, and the $s+1$ points $P, P_{1}, \ldots, P_{s}$ and $\Lambda_{2}$ onto a generic configuration

$$
P^{\prime} \cup P_{1}^{\prime} \cup \ldots \cup P_{s}^{\prime} \cup \Lambda_{2}^{\prime} \subset \Pi_{2} \text {. }
$$

Since in $\Pi_{2}$ the configuration above is non-feasible (see Proposition 4.1), then we conclude by Lemma 4.6 .

Case $s \geq n_{2}+3$, and $n_{1}=n_{2}$. Set $m=n_{1}=n_{2}$.

By projecting $\mathbb{P}^{n}$ from the linear spaces $\left\langle\Lambda_{2} \cup \ldots \cup \Lambda_{r}\right\rangle$ and $\left\langle\Lambda_{1} \cup \Lambda_{3} \cup \ldots \cup \Lambda_{r}\right\rangle$ onto $\Pi_{3} \simeq \mathbb{P}^{m}$, one sees that the images of the points $P_{i}$ give two sets of $s \geq m+3$ generic points in $\mathbb{P}^{m}$, which are not projectively equivalent, but they would be if $\Lambda$ were feasible.

Example 4.9. As a straightforward application of the previous Theorem, we have that the weight vector $(p, 1,1,0)$ is feasible if and only if $p \leq 5$.

\section{Applications}

5.1. Homogenous configurations. We can focus our attention on homogeneous configurations of linear spaces, i.e. configurations whose components all have the same dimension; notice that this is the setting of Veronese's Theorem 1.1 where only points are involved. In this simplified situation, Propositions 3.1 and 4.1 are enough to determine whether $\Lambda$ is feasible or non-feasible in almost all cases. More precisely, the following proposition shows that for $n \gg 0$ there is at most one case not covered.

Proposition 5.1. Let $n \geq 3$, and let $\Lambda \subset \mathbb{P}^{n}$ be a homogeneous configuration of $l$ generic $i$-dimensional linear spaces. If $n>i^{2}+5 i+1$, then $\Lambda$ is feasible for $l \leq \frac{n+3}{i+1}$, and $\Lambda$ is non-feasible for $l>\left\lceil\frac{n+3}{i+1}\right\rceil$.

Proof. By Propositions 3.1 and 4.1 we have only to consider the values of $l$ such that

$$
\frac{n+3}{i+1}<l \leq \frac{(n+3)(n-1)}{(i+1)(n-1-i)},
$$

as Proposition 3.1 gives feasibility for $l \leq \frac{n+3}{i+1}$ and Proposition 4.1 shows nonfeasibility for $l>\frac{(n+3)(n-1)}{(i+1)(n-1-i)}$.

It is easy to show that, for $n>i^{2}+5 i+1$, we have

$$
\frac{(n+3)(n-1)}{(i+1)(n-1-i)}-\frac{n+3}{i+1}<1 .
$$

Hence if $i+1$ divides $n+3$, then $l>\frac{(n+3)(n-1)}{(i+1)(n-1-i)}$ is equivalent to $l>\left\lceil\frac{n+3}{i+1}\right\rceil=\frac{n+3}{i+1}$, and the conclusion follows.

If $i+1$ does not divide $n+3$, let $q, r \in \mathbb{N}$ be such that

$$
n+3=q(i+1)+r, \quad 0<r<i+1 .
$$

By a direct computation we get

$$
\frac{(n+3)(n-1)}{(i+1)(n-1-i)}-q>1,
$$

hence by (3) we have 


$$
1+q<\frac{(n+3)(n-1)}{(i+1)(n-1-i)}<1+\frac{n+3}{i+1}=1+q+\frac{r}{i+1}
$$

then $l>\frac{(n+3)(n-1)}{(i+1)(n-1-i)}$ is equivalent to $l>q+1=\left\lceil\frac{n+3}{i+1}\right\rceil$, and we are done.

If the components of $\Lambda$ are all lines, we have an interesting result, which covers all cases except for $(n, l)=(5,5)$ and $(n, l)=(7,6)$, that is it is not know whether configurations of five generic lines in $\mathbb{P}^{5}$, or of six generic lines in $\mathbb{P}^{7}$ are feasible or not.

Proposition 5.2. Let $n \geq 3$, let $\Lambda \subset \mathbb{P}^{n}$ be the union of $l$ generic lines, then

i) $\Lambda$ is feasible in the following cases:

- $n=3$ and $l \leq 6$;

- $n>3$ and $l \leq\left\lfloor\frac{n}{2}\right\rfloor+2$;

ii) $\Lambda$ is non-feasible in the following cases:

- $n=3$ and $l \geq 7$;

- $n=5$ and $l \geq 6$;

- $n=7$ and $l \geq 7$;

- $n>3, n \neq 5, n \neq 7$ and $l \geq\left\lfloor\frac{n}{2}\right\rfloor+3$.

Proof. i) For $n=3$ see [Wak22], or [CC07, Proposition 3.1].

If $n>3$ is odd, by Proposition 3.1 we have that $\Lambda$ is feasible if $2 l \leq n+3$, that is for $l \leq\left\lfloor\frac{n}{2}\right\rfloor+2$.

If $n>3$ is even, by applying Theorem 4.8 with $s=4, r=\frac{n}{2}, n_{1}=\ldots=n_{t}=2$, we have that a generic configuration of $\frac{n}{2}$ lines and 4 points

$$
\Lambda_{1} \cup \ldots \cup \Lambda_{\frac{n}{2}} \cup\left\{P_{1}, \ldots, P_{4}\right\} \subset \mathbb{P}^{n}
$$

is feasible. Hence by choosing $P_{1}, P_{2}$ on a generic line and $P_{3}, P_{4}$ on another generic line, we get that $\Lambda$ is feasible for $l=\frac{n}{2}+2$, and so for $l \leq \frac{n}{2}+2$ also.

ii) For $n>7$, by Proposition 5.1 we immediately get the conclusion.

Let $n \leq 7$. By Proposition 4.1 we have that the configuration is non-feasible if $l>\frac{(n+3)(n-1)}{2(n-2)}$, so the conclusion easily follows for $n \neq 4$, or for $n=4$ and $l \geq 6$.

Finally for the case $(n, l)=(4,5)$, use the Bezout argument of Proposition 4.4 with $d=2$ and $k=1$. Notice that in this case it is crucial to have the knowledge of the Hilbert function of a generic set of lines, see for example [HH82].

5.2. One space in each dimension. Here we consider configurations of linear spaces having one component in each possible dimension.

Proposition 5.3. Consider the weight vector $L=(1, \ldots, 1)$ in $\mathbb{P}^{n}$. Then $L$ is feasible for $n \leq 5$ and non-feasible for $n \geq 8$.

Proof. We apply Propositions 3.1 and 4.1 to get that $L$ is feasible for

$$
n+3 \geq \sum_{i=0}^{n-2}(i+1)=\left(\begin{array}{l}
n \\
2
\end{array}\right)
$$

and $L$ is non-feasible for

$$
(n+3)(n-1)<\sum_{i=0}^{n-2}(i+1)(n-i-1)=\left(\begin{array}{c}
n+1 \\
3
\end{array}\right) .
$$

Solving these inequality we are left with the cases $5 \leq n \leq 7$. 
By applying Theorem 3.10 with $r=2, n_{1}=2$ and $n_{2}=3$ we get that the following generic configuration is feasible

$$
\Lambda_{1} \cup \Lambda_{2} \cup\left\{P_{1}, \ldots, P_{5}\right\} \subset \mathbb{P}^{5},
$$

where $\Lambda_{1}$ is a line, $\Lambda_{2}$ is a plane, and the $P_{i}$ are points. Hence by choosing $P_{1}, P_{2}, P_{3}, P_{4}$ on a generic linear space of dimension 3 , we get that $L=(1,1,1,1)$ is feasible in $\mathbb{P}^{5}$.

We notice again that, even in this simplified situation, there are cases which we can not solve.

5.3. The weight vectors $(\mathbf{0}, \mathbf{3}, \mathbf{0}, \ldots, \mathbf{0}, \mathbf{2}, \mathbf{0})$ are non-feasible. In this subsection we study configurations of three lines and two spaces of codimension 3 . In the particular case of $\mathbb{P}^{4}$, we have already proved the non-feasibility of five lines (see Proposition 5.2). The following proposition generalizes that result.

Proposition 5.4. The weight vector $L=(0,3,0, \ldots, 0,2,0)$ in $\mathbb{P}^{n}, n \geq 5$, is nonfeasible.

Proof. Let

$$
\Lambda_{L}=\Gamma_{1} \cup \Gamma_{2} \cup \Gamma_{3} \cup \Lambda_{1} \cup \Lambda_{2} \subset \mathbb{P}^{n}
$$

be a generic configuration of linear spaces of weight $L$, where $\Gamma_{i} \simeq \mathbb{P}^{1}, i=1,2,3$, and $\Lambda_{j} \simeq \mathbb{P}^{n-3}, j=1,2$, and let

$$
\Lambda^{\prime}=\Lambda_{L} \backslash \Gamma_{1}=\Gamma_{2} \cup \Gamma_{3} \cup \Lambda_{1} \cup \Lambda_{2} .
$$

By Proposition 4.4 we are done if we prove that there exists an integer $d$ such that

$$
\sum_{i=0}^{n-2} l_{i}(i+1)-1>d n
$$

and

Let $d=2$. Since:

$$
H\left(\Lambda_{L}, d\right)=H\left(\Lambda^{\prime}, d\right)+\left(\begin{array}{c}
d+1 \\
1
\end{array}\right)
$$

$$
\sum_{i=0}^{n-2} l_{i}(i+1)-1-d n=6+2(n-2)-1-2 n=1>0
$$

it suffices to show that a generic line imposes 3 independent conditions to the hyperquadrics containing $\Lambda^{\prime}$.

It is easy to prove that

$$
H\left(\Lambda_{1} \cup \Lambda_{2}, 2\right)=H\left(\Lambda_{1}, 2\right)+H\left(\Lambda_{2}, 2\right)-H\left(\Lambda_{1} \cap \Lambda_{2}, 2\right),
$$

so, since for $n=4,5$ we have $\Lambda_{1} \cap \Lambda_{2}=\emptyset$, and for $n>5$ we have $\Lambda_{1} \cap \Lambda_{2} \simeq \mathbb{P}^{n-6}$, we get

$$
H\left(\Lambda_{1} \cup \Lambda_{2}, 2\right)=2\left(\begin{array}{c}
n-3+2 \\
2
\end{array}\right)-\left(\begin{array}{c}
n-6+2 \\
2
\end{array}\right)=\frac{n^{2}+3 n-16}{2} .
$$

Now we expect that three generic lines impose 9 conditions to the hyperquadrics containing $\Lambda_{1} \cap \Lambda_{2}$, in other words, we expect that the Hilbert function of $\Lambda_{L}$ in degree 2 is

$$
\text { expected } H\left(\Lambda_{L}, 2\right)=9+\frac{n^{2}+3 n-16}{2}=\left(\begin{array}{c}
n+2 \\
2
\end{array}\right) .
$$


So, if we prove that there are not forms of degree 2 in the ideal $I_{\Lambda_{L}}$, then it follows that there are exactly three forms of degree two in $\left(I_{\Lambda^{\prime}}\right)_{2}$, and we are done.

We work by induction on $n$.

For $n=5$, first specialize the three lines $\Gamma_{i}$ onto a hyperplane $H=\{f=0\}$,

Consider the exact sequence,

$$
0 \longrightarrow\left(I_{\Lambda_{L} \backslash H}\right)_{1} \longrightarrow\left(I_{\Lambda_{L}}\right)_{2} \longrightarrow\left(I_{\Lambda_{L} \cap H}\right)_{2}
$$

where the first map is given by the multiplication by $f$, and where $\Lambda_{L} \backslash H$ is the residual of $\Lambda_{L}$ with respect to $H$, and $\Lambda_{L} \cap H$ is the trace of $\Lambda_{L}$ on $H$.

Since $\Lambda_{L} \backslash H=\Lambda_{1} \cup \Lambda_{2}$, and it does not lie on a hyperplane, then $\operatorname{dim}\left(I_{\Lambda_{L} \backslash H}\right)_{1}=$ 0 . Moreover, since $\Lambda_{L} \cap H$ consists of five lines in $\mathbb{P}^{4}$, we get $\operatorname{dim}\left(I_{\Lambda_{L} \cap H}\right)_{2}=0$ (see [HH82]). The conclusion follows from the semicontinuity of the Hilbert function.

Now assume $n>5$ and let $H=\{f=0\}$ be a hyperplane containing the three lines $\Gamma_{i}$. Consider the following exact sequence analogous to the one above,

$$
0 \longrightarrow\left(I_{\Lambda_{L} \backslash H}\right)_{1} \longrightarrow\left(I_{\Lambda_{L}}\right)_{2} \longrightarrow\left(I_{\Lambda_{L} \cap H}\right)_{2}
$$

Since $\Lambda_{L} \backslash H=\Lambda_{1} \cup \Lambda_{2}$ does not lie on a hyperplane, then $\operatorname{dim}\left(I_{\Lambda_{L} \backslash H}\right)_{1}=0$. Moreover $\operatorname{dim}\left(I_{\Lambda_{L} \cap H}\right)_{2}=0$ follows by the inductive hypothesis, so we get $\operatorname{dim}\left(I_{\Lambda_{L}}\right)_{2}=0$ and we are done.

5.4. A new result about defectiveness of higher secant varieties of SegreVeronese varieties. As noted in Remark 3.12 the existence of a suitable rnc yields that the Segre-Veronese variety $\mathbb{P}^{2} \times \mathbb{P}^{3}$ embedded using forms of bidegree $(1,2)$ is 5 -defective. By Theorem 3.10 we get the following new result involving Segre-Veronese varieties, for more on these varieties see [CGG05, CGG07].

Corollary 5.5. Let $V \subset \mathbb{P}^{N}$ be the Segre-Veronese variety $\mathbb{P}^{1} \times \mathbb{P}^{m} \times \mathbb{P}^{m}$ embedded with multi-degree $(2,1,1)$. Let $V^{s}$ be the $s^{\text {th }}$ higher secant variety of $V$, that is the closure of the union of all linear spaces spanned by s independent points of $V$. If

$$
m+2 \leq s \leq 2 m+1,
$$

then $V$ is s-defective, i.e. $V^{s}$ has not the expected dimension.

Proof. For $\left\lceil\frac{3(m+1)}{2}\right\rceil \leq s \leq 2 m+1$ we already know that $V$ is $s$-defective (see [CGG05, Section 3]). In fact the expected dimension of $V^{s}$ is $N$, but there exist form $f_{1}$ and $f_{2}$ of multidegree $(1,1,0)$ and $(1,0,1)$, respectively, passing through $s$ generic (simple) points, hence $f_{1} f_{2}$ is a form of multidegree $(2,1,1)$ through $s$ generic 2-fat points, which was not supposed to exist.

The case $m+2 \leq s \leq\left\lfloor\frac{3(m+1)}{2}\right\rfloor$ was not previously known. For such $s$ the expected dimension of $V^{s}$ is

$$
\exp \operatorname{dim} V^{s}=N-s(2 m+2) \text {. }
$$

Now

$$
\operatorname{dim} V^{s}=\operatorname{dim}\left(I_{W \cup Z}\right)_{4},
$$

where $W$ is a generic subscheme of $\mathbb{P}^{2 m+1}$ consisting of one double point $Q$ and two triple linear spaces $\Lambda_{1}, \Lambda_{2}$ of dimension $m-1$, while $Z$ consists of $s$ generic double points $P_{1}, \ldots, P_{s}$ (see [CGG05, Theorem 1.5]). Since $\operatorname{dim}\left(I_{W}\right)_{4}=N=3(m+1)^{2}$, we have that

$$
\operatorname{dim}\left(I_{W \cup Z}\right)_{4}=N-h
$$

where $h$ is the number of independent conditions imposed by the $s$ double points $P_{i}$ to the forms of $\left(I_{W}\right)_{4}$. Note that the expected value for $h$ is $s(2 m+2)$. 
By Theorem 3.7 we know that there exists an $\operatorname{rnc} \mathcal{C}$ maximally intersecting the configuration $\Lambda_{1} \cup \Lambda_{2} \cup Q \cup P_{1} \cup \cdots \cup P_{s}$. Let $F \in\left(I_{W \cup Z^{\prime}}\right)_{4}$, where $Z^{\prime}$ consists of the $s-1$ double points $P_{1}, \ldots, P_{s-1}$ and the simple point $P_{s}$. We have

$$
\text { degree }\{F=0\} \cap \mathcal{C} \geq 6 m+2 s+1 \geq 6 m+2(m+2)+1=8 m+5,
$$

so by Bezout Theorem $\mathcal{C} \subset\{F=0\}$. Hence the double point $P_{s}$ imposes less then $2 m+2$ conditions to the forms of $\left(I_{W \cup Z}\right)_{4}$, so

$$
\operatorname{dim} V^{s}=\operatorname{dim}\left(I_{W \cup Z}\right)_{4}=N-h>N-s(2 m+2)=\exp \operatorname{dim} V^{s},
$$

and the conclusion follows.

\section{REFERENCES}

[Bab33] D. W. Babbage. The number of rational quartic curves with seven assigned trisecant planes in space of four dimensions. Jour. Lon. Math. Soc, 8:9-11, 1933.

[BPS05] Anders Björner, Irena Peeva, and Jessica Sidman. Subspace arrangements defined by products of linear forms. J. London Math. Soc. (2), 71(2):273-288, 2005.

[CC07] E. Carlini and M. V. Catalisano. Existence results for rational normal curves. J. Lond. Math. Soc. (2), 76:73-86, 2007.

[CGG05] M. V. Catalisano, A. V. Geramita, and A. Gimigliano. Higher secant varieties of SegreVeronese varieties. In Projective varieties with unexpected properties, pages 81-107. Walter de Gruyter GmbH \& Co. KG, Berlin, 2005.

[CGG07] M. V. Catalisano, A. V. Geramita, and A. Gimigliano. Segre-Veronese embeddings of $\mathbb{P}^{1} \times \mathbb{P}^{1} \times \mathbb{P}^{1}$ and their secant varieties. Collect. Math., 58(1):1-24, 2007.

[Ci101] C. Ciliberto. Geometric aspects of polynomial interpolation in more variables and of Waring's problem. In European Congress of Mathematics, Vol. I (Barcelona, 2000), volume 201 of Progr. Math., pages 289-316. Birkhäuser, Basel, 2001.

[Deb08] Gery Debongnie. Rational homotopy type of subspace arrangements with a geometric lattice. Proc. Amer. Math. Soc., 136(6):2245-2252, 2008.

[Der07] Harm Derksen. Hilbert series of subspace arrangements. J. Pure Appl. Algebra, 209(1):91-98, 2007.

[EHP03] David Eisenbud, Klaus Hulek, and Sorin Popescu. A note on the intersection of Veronese surfaces. In Commutative algebra, singularities and computer algebra (Sinaia, 2002), volume 115 of NATO Sci. Ser. II Math. Phys. Chem., pages 127-139. Kluwer Acad. Publ., Dordrecht, 2003.

[Ger96] A. V. Geramita. Inverse systems of fat points: Waring's problem, secant varieties of Veronese varieties and parameter spaces for Gorenstein ideals. In The Curves Seminar at Queen's, Vol. X (Kingston, ON, 1995), volume 102 of Queen's Papers in Pure and Appl. Math., pages 2-114. Queen's Univ., Kingston, ON, 1996.

[GR03] T Graber and K. Ranestad. Veronese surfaces and line arrangements. MSRI Preprint \#2003-017, 2003.

[Har92] J Harris. Algebraic geometry, A first course. Graduate Texts in Math. Springer-Verlag, New York, 1992.

[HH82] Robin Hartshorne and André Hirschowitz. Droites en position générale dans l'espace projectif. In Algebraic geometry (La Rábida, 1981), volume 961 of Lecture Notes in Math., pages 169-188. Springer, Berlin, 1982.

[Kap93] M. M. Kapranov. Veronese curves and Grothendieck-Knudsen moduli space $\bar{M}_{0, n} . J$. Algebraic Geom., 2(2):239-262, 1993.

[Roo38] T. G. Room. The Geometry of Determinantal Loci. Cambridge University Press, 1938.

[Sid07] Jessica Sidman. Resolutions and subspace arrangements. In Syzygies and Hilbert functions, volume 254 of Lect. Notes Pure Appl. Math., pages 249-265. Chapman \& Hall/CRC, Boca Raton, FL, 2007.

[Ver82] G. Veronese. Behandlung der projectivischen verhältnisse der räume von verschiedenen... Math. ann., XIX:161-234, 1882.

[Wak22] E. K. Wakeford. Chords of twisted cubics. Proc. Lond. Math. Soc., 21:98-113, 1922. 
[Zak93] F. L. Zak. Tangents and secants of algebraic varieties, volume 127 of Translations of Mathematical Monographs. American Mathematical Society, Providence, RI, 1993. Translated from the Russian manuscript by the author.

Enrico Carlini, Dipartimento di Matematica del Politecnico di Torino, Corso Duca Degli Abruzzi 24, 10129 Turin, Italy.

E-mail address: enrico.carlini@polito.it

Maria Virginia Catalisano, Diptem - Dipartimento di Ingegneria della Produzione, Termoenergetica e Modelli Matematici, Piazzale Kennedy, pad. D 16129 Genoa, Italy.

E-mail address: catalisano@dipem.unige.it 